\title{
Schneider, Laura: Measuring global media freedom. The Media Freedom Analyzer as a new assessment tool
}

\author{
Wiesbaden: Springer VS 2020. 252 Seiten. Preis: $€$ 50,28 (e-book)
}

\author{
Melanie Radue
}

Online publiziert: 30 . September 2020

(C) Der/die Autor(en) 2020

Bislang stützen sich wissenschaftliche Studien zur Messung von Medienfreiheit meist auf die Ergebnisse der Rankings von internationalen Nichtregierungsorganisationen wie Freedom House (FH), International Research \& Exchanges Board (IREX) und Reporters Without Borders (RWB). Trotz methodischer Mängel und perspektivischer Verzerrung sind vor allem die Rankings von FH und RWB weltweit Grundlage für Analysen und Entscheidungen in Medien, Politik und Wissenschaft. Die geringe wissenschaftliche Qualität der Ergebnisse der viel zitierten Rankings nimmt Laura Schneider zum Anlass, in ihrer Dissertationsschrift ein erstes empirisch validiertes Instrument zum Vergleich von Medienfreiheit vorzustellen.

Das Buch unterteilt sich in einen theoretischen und einen empirischen Teil und entfaltet sich entlang drei zentraler Fragestellungen im wissenschaftlichen Diskurs zum Thema (S. 3): „1. What are the challenges of international media freedom measurement? 2. What are the main differences regarding the concept of media freedom between world regions? 3. How should an improved international media freedom measure be comprised?"

Der theoretische Teil trägt der Problematik der konzeptionellen Unschärfe von Medienfreiheit Rechnung. Zunächst stellt die Autorin verschiedene Konzepte und theoretische Grundlagen zu Medienfreiheit vor, um sich dann auf die Liberal Theory und die Social Responsibility Theory von Siebert, Peterson und Schramm (1956) als Ausgangspunkt für die eigene Untersuchung zu fokussieren. Schneider begründet die Relevanz der normativen Theorien für ihre eigene Arbeit über die Verankerung in Grundgesetzen und Menschenrechtsabkommen in weiten Teilen der Welt. Wichtig ist hier, dass Schneider die theoretische Universalität der global verbreiteten Normen

Dr. M. Radue $(\bowtie)$

Lehrstuhl für Kommunikationswissenschaft, Friedrich-Alexander-Universität Erlangen-Nürnberg,

Findelgasse 7/9, 90402 Nürnberg, Deutschland

E-Mail: melanie.radue@fau.de 
ebenso beleuchtet wie ihre unterschiedlichen regionalen Adaptionen und die damit verbundene Ambiguität zum Konstrukt und Verständnis von Medienfreiheit.

Im Kontext eines Überblicks zum aktuellen Forschungsstand zur Messung von Medienfreiheit erläutert Schneider, wieso internationale Vergleiche wichtig sind und welchen Einfluss sie in Politik, Medien und Wissenschaft haben. Die Autorin stellt dazu einschlägige Messinstrumente und die aktuell relevantesten Rankings von FH, RWB, IREX, Friedrich-Ebert-Stiftung und UNESCO vor. Die Autorin hebt den Mehrwert der Indizes als Institutionen hervor, die Veränderungen von Medienfreiheit auf globaler Ebene vergleichen und der Öffentlichkeit zur Verfügung stellen. Die kritische Auseinandersetzung mit der Methodik dieser fünf Medienfreiheitsindizes bestärkt jedoch Schneiders Argument für die Entwicklung eines neuen Messinstruments.

Der zweite Teil der Dissertationsschrift erläutert sehr systematisch und klar alle Forschungsschritte, die zum Entwurf des Media Freedom Analyzer (MFA) führen. Der MFA soll das komplexe und multidimensionale Konstrukt Medienfreiheit in einer simplifizierten Form messen. Er folgt dabei einem pragmatischen anwendungsund politikorientierten Ansatz.

Basierend auf der Analyse der fünf Medienfreiheitsindices, einer eingehenden Literatursichtung und Überlegungen der Autorin auf Grundlage vorheriger Forschung entsteht ein Set von 47 potentiellen Indikatoren für den MFA, die in 7 Dimensionen eingeteilt sind: Safety and Security, Independence of the Media, Legal Conditions, Structural Conditions, Participation, Professionalism, Diversity and Quality of Media Products. Die Wichtigkeit dieser 47 Indikatoren wird in einer Online-Umfrage mit 946 Medienfreiheits-Expert*innen aus 126 Ländern, die alle Weltregionen repräsentieren, abgefragt. Alle 47 Indikatoren werden von den Expert*innen als wichtig eingestuft. Auf dieser Grundlage bestätigt die Autorin, dass, zumindest aus normativer Perspektive, Medienfreiheit weltweit als ein globales Menschenrecht angesehen wird und ein breites gemeinsames Verständnis davon besteht. Nichtsdestotrotz deuten signifikante kulturelle Unterschiede zwischen verschiedenen Weltregionen darauf hin, dass es keinen Konsens zu den Details des Konzepts Medienfreiheit gibt.

Mit den aufgezeigten kulturellen Differenzen bestätigt sich die oft geäußerte Kritik an westlich-ideologisch verzerrten Medienfreiheitsindizes. Schneider schlussfolgert an dieser Stelle, dass die Berücksichtigung der Perspektiven von Expert*innen aus allen Teilen der Welt in der Bewertung der von ihr gewählten Indikatoren eine solche Verzerrung bereinigt. Jedoch beruht bereits die Auswahl der Indikatoren vorwiegend auf den gleichen westlich-normativen Ansätzen, die auch den anderen von ihr kritisierten Messinstrumenten zugrunde liegen. So werden bspw. in den Indikatoren Einflüsse von ökonomischen und politischen Akteuren, wie auch religiöse Einflussnahmen berücksichtigt. Andere relevante Akteure wie das Militär (u.a. in Thailand und Myanmar) oder Monarchen (u.a. in Malaysia und Thailand) finden sich allerdings nicht wieder. Damit liefert die Studie zwar ein sehr gut recherchiertes, transparentes und empirisch geprüftes Tool, welches erstmals eine auf Expert*innenmeinung beruhende Gewichtung der verschiedenen Indikatoren zur Messung von Medienfreiheit liefert. Sie geht jedoch wenig über bereits Bekanntes hinaus. Ergänzende relevante Faktoren ergeben sich sicherlich aus empirischen Er- 
gebnissen zu Einflussfaktoren in anderen Weltregionen. Insbesondere die westlichnormative theoretische Perspektive hätte erweitert werden können. Ebenso würde ein induktives Vorgehen - wie Schneider es auch selbst in der Arbeit reflektiert der bestehenden Verzerrung Rechnung tragen.

Daraus ergibt sich der größte Kritikpunkt an einer solch lang ersehnten Publikation zur Messung von Medienfreiheit: das Festhalten an westlich-normativ verzerrten Ansätzen. Hierdurch bleibt die Möglichkeit der aufwendigen Studie, kontextualisierte, nicht-eurozentrische Perspektiven zu integrieren, ungenutzt. Dennoch durchdringt Schneider alle problematischen Aspekte des Themas und entwickelt ein relevantes Tool, das eine Korrektur der bestehenden Indizes bieten kann. Damit hat es eine hohe Relevanz für alle, die sich kritisch mit internationalen Vergleichen und Messungen von Medienfreiheit befassen.

Funding Open Access funding enabled and organized by Projekt DEAL.

Open Access Dieser Artikel wird unter der Creative Commons Namensnennung 4.0 International Lizenz veröffentlicht, welche die Nutzung, Vervielfältigung, Bearbeitung, Verbreitung und Wiedergabe in jeglichem Medium und Format erlaubt, sofern Sie den/die ursprünglichen Autor(en) und die Quelle ordnungsgemäß nennen, einen Link zur Creative Commons Lizenz beifügen und angeben, ob Änderungen vorgenommen wurden.

Die in diesem Artikel enthaltenen Bilder und sonstiges Drittmaterial unterliegen ebenfalls der genannten Creative Commons Lizenz, sofern sich aus der Abbildungslegende nichts anderes ergibt. Sofern das betreffende Material nicht unter der genannten Creative Commons Lizenz steht und die betreffende Handlung nicht nach gesetzlichen Vorschriften erlaubt ist, ist für die oben aufgeführten Weiterverwendungen des Materials die Einwilligung des jeweiligen Rechteinhabers einzuholen.

Weitere Details zur Lizenz entnehmen Sie bitte der Lizenzinformation auf http://creativecommons.org/ licenses/by/4.0/deed.de.

Dr. Melanie Radue ist wissenschaftliche Mitarbeiterin am Lehrstuhl für Kommunikationswissenschaft an der Friedrich-Alexander-Universität Erlangen-Nürnberg. 